\title{
The distinctive character of human being in evolution
}

\author{
DANIEL TURBÓN \\ University of Barcelona \\ turbon@ub.edu \\ ORCID: 0000-0002-4782-6657
}

\begin{abstract}
Human beings, as we know and understand them today, are the result of a lengthy, two million year old process that has made them one of the most powerful and beautiful biological beings. The process of encephalisation in humans, combined with the development of areas of speech, brought about by a neurological reorganisation that may have taken place before the increase in brain size, has enabled humanity to generate a tremendous cognitive capacity that in turn has led to the development of what we know of as culture. Culture influences biological development. No other species has achieved the like anywhere. For human survival, culture is a new dimension, a new habitat, which humanity has to adapt as it creates it. Culture is not written into the genome, but it can be transmitted and communicated thanks to speech. This enables knowledge to be shared and transmitted to other members of the group or society, to communicate ideas, concepts and abstractions. Knowledge enables a society to form a structure and make it more complex than a simple agglomeration of individuals while also creating an environment where raising children is viable since it can guarantee their survival, giving them the right treatment to enable them to reach adulthood. It has been said that 'humanity is a spirit in time', hence the need to understand the essence of human natural history; the importance of paying it sufficient attention to ensure that what is attractive about its history does not become indigestible.
\end{abstract}

Keywords: adaptation and adaptability; encephalization; speech; human ontogeny; selfawareness. 


\section{Intoduction. Present-Day Humans Have Equivalent Intellectual Potential}

Modern humans potentially have similar intellectual capacities, although these are expressed in different manners. The origins and development of the powerful human mind is necessarily prior to the dispersion of modern humans around our planet, which is which is gradually being established by the comparative analysis of different biological clocks. The concept of prehistoric man as someone stupid and ignorant, which took shape within the context of the slave-owning societies of three centuries ago, has been owercome, in line with archaeological discoveries. From the 18th c. onwards, an updated version of the Aristotelian concept called The Great Chain of Being, placed some peoples and societies on a lower rung, below other humans In 1877 the book "Ancient Society", written by the American anthropologist Lewis H. Morgan, developed the theory that Humanity had developed socially. although unevenly, in three stages of human progress, like steps on a staircase: Savagery, Barbarian and Civilization, similar to the progress of a childlike mentality towards adulthood. This idea influences many thinkers such as K. Marx, F. Engels and was quoted by others such as C.R. Darwin. In the 20th century this point of view changed, thanks to filed studies conducted by people such as B. Malinowsky (1926) and, above all, by the work of C. Lévi-Strauss (1949), who considered that there were profound differences between primitive thought and children's thinking. The fact is that so called 'primitive culture', even with its lower level of development, is an adult one, and totally incompatible with the manifestations of childhood to be found in a more technologically advanced society.

However, the Theory of Evolution has been used to justify domination and inequality, a very different concept from that of diversity, slavery and colonialism, and the scientific racism arising from the eugenic thinking initiated by Francis Galton (1822-1911). Biological determinism set out to prove that shared norms of behaviours such as social and economic differences between groups, basically differences of race, class and gender, arise from certain innate hereditary differences, and that in this regard, 
society is a faithful mirror image of biology. In other words, the behaviour of animals and human beings, has obeyed the dictates of the survival of their genes, and has extended to complex social systems that are adapted to the most favourable evolutionary process. This 'naturalistic fallacy', based on an exaggerated interpretation of the concept of 'natural selection' has since been disproved by Epigenetics.

Genes are expressed within an environment, especially prenatal development, and are continuously being controlled by the environment via mechanisms such as epigenetics, which is commonly defined as: "stably heritable phenotype resulting from changes in a chromosome without alterations in the DNA sequence". The field of Epigenetics is discovering a new reading of the genome (epigenome). The term 'epigenetic modification' is understood as referring to all those changes undergone by the chromatin but that do not affect the nucleotide sequence: that is why they are not "genetic" modifications, but rather changes that are "above" (which is the meaning of epi in Greek). In recent years, experiments have demonstrated the major importance of mechanisms that regulate the activity of chromatin activity via epigenetic changes.

It is very important to understand that the structural and functional aspects of the Human Genome form part of a "dynamic" model, in which a chromatin region will be in a state that favours gene expression to a greater or lesser extent depending on the type of sequences that make it up and also on the epigenetic modifications at such a level. Therefore, the epigenome varies a great deal from one cell to another: cells that have a similar genome express different genes and likewise perform different functions. Our own experiences can mark our genetic material in ways unknown up till now, and such marks can be transmitted to future generations.

Dobzhansky criticised Social Darwinism and racism, commenting on the limitations of 'natural selection' $(1962,11)$ :

Natural selection is simply the antonym of artificial selection [...] Who is the "fittest" in the evolutionary "survival of the fittest" is a most complex matter which has not been fully clarified even yet. One thing which is clear is that 
the fittest is not necessarily a romantic figure, or a victorious conqueror, or a superman. He is most likely to be merely a prolific parent.

About 2,500 years have gone by since the caste system in India sealed off the reproductive system. Although modern India has a long way to go before it can abolish all the inequalities of rank and opportunities, all the castes have produced individuals with sufficient capacity to acquire a non-traditional education and engage in non-traditional activities. In much the same way, the revolutionary overthrow of old elites, which were physically annihilated or forced to migrate, were genetic experiments on a smaller scale. Gratuitous predictions were made declaring that such decapitations would lead to a scarcity of talent. The example of Russia has shown that the prediction was a mistaken one, since persons of talent recruited from the old inferior classes, which had not had the chance to stand out in any way, have created an intellectual class that is by no means inferior to the old one.

\section{The Essentials of the Evolution of Humankind}

How, therefore, can the different technological and scientific output of different modern peoples be explained? Firstly by understanding the role of genes in human evolution. It is useless to seek the genes that cause bodily decoration, etiquette, incest taboos, figures or the concept of the soul. Such genes do not exist. Genes transmit patterns of biological development, the outcome of which is subject to the environment. Human genes have achieved something that other genes could not do: form the basis of an extra-somatic culture, which turned out to be a more effective method for adapting to the environment than that used by any other species. Human cultural development cannot be found in biological nature. From a biological perspective, human culture stands out from other animal species because of the enormous investment in social learning, the result of which is an enormous accumulation of information stored outside the body. A more complex technical and cultural social organisation has arisen from a more complex brain, and that in turn has enabled further development of the brain, etc., meaning that the brain is in constant evolution. 
Knowledge can be shared and transmitted to other members of the group or society, thereby permitting others to learn, communicate ideas, concepts or abstractions. Thus an environment was created where raising children is viable since it can guarantee their survival and so enable them to reach adulthood.

The second factor in technological achievements or progress can be found in the History of Populations. As Culture is not determined by genes, peoples can become poor as a result of wars or catastrophes, and so lose their cultural baggage. Civilisations have had moments when they were at their apogee and others when they were in a state of collapse. As regards societies such as the hunter gatherers (once called 'savages'), there are evident factors that limit their cultural development because they have adapted to an adverse environment that exerts selective pressures on population size, which is limited and fluctuating, and which forces people to obtain resources all day long in order to survive. This state of affairs impedes their potential demographic, technological, economic and cultural development.

If all modern humans have similar capacities, when did their geographical dispersion throughout the continents take place? Studies of the diversity of DNA of current populations have made it possible to establish, using molecular clocks, that the Khoi-San of the Kalahari separated from other modern Africans more than 100 kya ago (Schlebusch et al. 2012). What is more, the polymorphisms of the Y chromosome, an indicator of masculine diversity, show an age of $330 \mathrm{ky}$. (confidence interval of 95\% 1/4 237-581 kya). The calculations of the molecular clock of the Y chromosome give older dates than current estimates of mytochondrial DNA, a marker of female lineages (Figure 1) along with the dating of older anatomically modern human fossils.

On the other hand, it is now beyond doubt that the Neanderthals interbred with modern humans in some areas, which indicates that they were not genetically different species (Green et al 2010, Reich et al. 2011, Sankararaman et al. 2012). As for the psychological nature of this now extinguished human lineage, the increased number of sites with good evidence of fire throughout the Late Pleistocene shows that European 
Neanderthals had fire management not unlike the one documented for Upper Palaeolithic groups (Roebroeks \& Vila 2011). One of the best indicators of human intellect is the use of fire. In addition, the earliest known personal ornaments come from the Middle Stone Age in southern Africa, c. 75,000 years ago, and are associated with anatomically modern humans. In Europe, such items are not recorded until after 45,000 radiocarbon years ago, in Neanderthal-associated contexts that significantly predate the earliest evidence, archaeological or paleontological, for the immigration of modern humans. These temporal patterns suggest that the emergence of "behavioural modernity" was triggered by demographic and social processes and is not a speciesspecific phenomenon; a corollary of these conclusions is that the corresponding genetic and cognitive basis must have been present in the genus Homo before the evolutionary split between the Neanderthal and modern human lineages (Zilhao 2007).

\section{The Evolution of the Language and the Brain}

\subsection{Adaptation and Adaptability}

According to Tobias (1999) the brain-size increase process that took place in early Homo was a challenge to survival, about 2 million years ago (mya). It was possible because a series of conditions coincided as had never happened before. These first humans exploited a cultural econiche, transmitting the knowledge required for survival to their increasingly dependent young, by means of articulated language. There was a feedback loop because, as the young became increasingly encephalized, they were also more vulnerable, took longer to mature and if they were looked after until they reached reproductive age, the situation repeated itself and so on. Finally, the fact that extinction did not take place in the light of so much dependency means that the feedback mechanism was successful (Figure 2).

If we assume that encephalization improves adaptative abilities, understood as "greater evolutive flexibility", we should consider the difference between adaptation and adaptability, in the evolutive - that is genetic - 
sense of these terms. Is it an advantage to be more encephalized? Adaptation and adaptability, in evolution, are inversely proportional: the better adapted an animal is to its environment, the less plasticity will it retain to adapt to a new environment should conditions change. However, in the case of the Homo genus, the use of Culture enabled human beings to reverse this relation by combining adaptation with adaptability (Tobias 1997). There is no more powerful force in Nature than the ability to promote adaptation by greatly broadening the evolutive flexibility of its possessors.

In summary, the progression from Biological Evolution to Cultural Evolution endowed Homo with a greater ability to respond to the challenges of the environment and with greater flexibility, as Culture is a product free from genetic influence that evolves too quickly to be subjected in any way to the mechanism of natural selection.

\subsection{Brain Reorganization and Allometry in Hominids}

A major reorganizational change from a pongid to hominid pattern occurred some 3-4 million years ago in the posterior parietal, anterior occipital, and superior temporal portions of the cerebral cortex (Figure 3). This occurred before frontal lobe (Broca's area) reorganization in Homo habilis, about 1.8 million years ago. The doubling of brain size from roughly $750 \mathrm{cc}$ in $H$. habilis to $1400 \mathrm{cc}$ in modern $H$. sapiens occurred after (at least) three major reorganizational changes evolved in earlier hominid brains (Figure 4).

According to Holloway (1995), these were:

1. Reduction of the primary visual striate cortex (Brodmann's area 17) and relative enlargement of the extrastriate parietal cortex (areas 18 and 19), the angular gyrus (area 39), and the supramarginal gyrus (area 40) of the inferior parietal lobe. This represents the first major change from pongid to human brain organization and occurred between 3 and 4 million years ago.

2. A reorganization of the frontal lobe, mainly involving the third inferior frontal convolution known as Broca's area, towards a definitively human external morphology most fully in evidence in the KNM- 
ER1470 specimen of $H$. habilis. This probably occurred between 2.5 and 1.8 million years ago.

3. In addition to brain enlargement (mostly as an allometric relation to body size), there was a change from a pongid pattern of minimal cerebral asymmetries to a human pattern involving a higher degree of left-occipital and right-frontal petalial enlargements, associated with greater hemispheric specialization. This appears most clearly in H. habilis, but may have occurred earlier in Australopithecus africanus, 2 to 3 million years ago.

Regarding brain volume changes integrated with reorganization, Holloway (1995) suggests the following sequence:

1. There was a small allometric increase in brain size from early A. afarensis to later $A$. africanus, i.e. from roughly $400 \mathrm{cc}$ to perhaps $460 \mathrm{cc}$.

2. From A. africanus to Homo habilis there was an increase in brain size from about 450 cc to 700-750 cc and in H. habilis, an increase of 250-300 cc. As there is little solid evidence for any major increase in body size between the two species, we cannot be certain that this significant increase was allometrically related to body size. (That is, specimens of habilines such as KNM-ER 1813 and KNM-ER 1805, or $\mathrm{OH}$ 7, or $\mathrm{OH} 8$, were surely smaller than KNM-ER 1470 in both brain and body size.)

3. From $H$. habilis to $H$. erectus, there was a modest increase of brain size from roughly $750 \mathrm{cc}$ to perhaps $900 \mathrm{cc}$, which was most likely an allometric one, as the KNM-WT 15000 'strapping youth' postcranial elements suggest a significant increase in body size, but only a minor increase in brain size.

The backward expansion of the parietal lobe of the brain, that is, of Brodmann's association areas 39 and 40, was progressively formed from the first australopithecines. Such cerebral areas are of crucial importance in the elaboration of our symbolic behavior, since they process the non-emotional nervous impulses, unlike the rest of the animals (Figure 5). The main feature that allows to place the mentioned areas in the cranial molds is the sulcus lunatus, a transverse groove located at the end of the intraparietal 
sulcus, which divides the parietal lobe into two parts. The sulcus lunatus is visible in all primates except humans, in which, because it has receded, it is almost hidden in the back of the brain, which is why it is not usually seen in handbooks of human anatomy.

In Hadar's Australopithecus afarensis it is presented in a posterior position, an indication that 3,5 million years ago the reorganization of the brain in the human line had begun. The South African specimen Stw 505 of Sterkfontein shows an unmistakable posterior position of the sulcus lunatus, confirming that the brain of Australopithecus africanus had already reorganized neurologically, at the latest, with the appearance of this taxon.

According to Holloway, the causes that originated the reorganization of the posteriori parietal cortex would be associated with the increase in social complexity, the use of tools and the need for communication. The language is closely associated with the manufacture of stone tools by early hominids (Holloway 1995, 2009).

Many archeologists and paleoanthropologists believe that there could not have been any hominids with language until one finds evidence for symbolic art, as found in the caves of France and Spain, dating to roughly 20 000-30 000 years ago. Others, including this author, conclude that if one finds evidence for cerebral asymmetries, including those for left/right Broca's regions, then one must consider that language could well have been present, at least by $1.8 \mathrm{Ma}$, since the fossil hominid (see Holloway 2009, 356).

\subsection{Speech and the Foxp2 regulatory gene}

The process of encephalisation in humans, linked to the development of language areas caused by a neurological reorganisation that perhaps took place before the increase in brain size, enabled cognitive capacities to be generated in the human lineage, which in turn permitted the creation of what we understand as culture. This culture is not written into the genome but it can be communicated and transmitted thanks to speech. The advantage of spoken language is that knowledge is shared and transmitted to other members of the group or society and so enable others to learn, 
communicate ideas, concepts and abstract elements. Humans can adapt to the environment but at the same time language also enables them to adapt to new situations that arise. This adaptability would not be possible without spoken language, which gives fundamental importance to the use of tools and their construction and linguistic communication. Therefore, spoken language has become a necessary condition for our development since the first representatives of Homo walked on the planet.

In a spoken language, genetic endowment is a necessary precondition, but it is not sufficient to expand and grow an individual's linguistic capacity. It is universal, given that every baby born without alterations possesses it from the moment of birth, or even before. It is specific because it is exclusive to the human species, at least in the strict sense of the word. Syntax is a computational system. Sign languages also show this characteristic.

Children learn to speak very early in life, spontaneously and with no apparent effort, considering that the stimuli received are very limited, heterogeneous, incomplete, mistaken and contradictory. In fact, parents do teach their children to speak, what they do is provide them with stimuli and possibilities for communication and relationships, as well as evidence for setting parameters. But the elements of Universal Grammar are already programmed into the individual at genetic level: no one taught us to transfer elements of the phrase, syntax and abstract concepts.

In the nineteen nineties a gene, Foxp2, was found to be related to human language, because mutations of the gene produced language disorders. In 2001 these disorders were studied in a number of generations in an English family called KE, whose members had difficulties in speaking and articulating words and committed basic grammatical errors over several generations. $50 \%$ of the members of the KE family presented a severe language-related disorder. The family members that were affected were unable make coordinated movements of the tongue and lips necessary for speaking clearly (verbal dyspraxia). Those affected, without being deaf, were unable to gesture or make the movements of the mouth essential for pronouncing any phrase. Members of the family also presented symptoms of dyslexia and grammatical difficulties that stopped them from writing correctly (Fisher 
\& Scharff 2009). This discovery is undeniable proof of the genetic basis of human speech, and appears in the cerebral regions related to speech and language from the moment the embryo starts to develop. Foxp2 in humans is located in chromosome 7. Foxp2 operates on a wide variety of genes from a child's development stage onwards (Bolhuis et al. 2010).

The repercussions of Fopxp2 for evolutionary studies are no less important. The notion that Neanderthals could not speak or had speech defects has now been ruled out. An analysis of Neanderthal DNA indicates that they shared a form of the FOXP2 gene with living humans, the absence of which impairs speech and cognitive processing related to human language (Trinkaus 2007).

At the same time, Foxp2 is not uniquely human. It exists in a wide variety of species, where it regulates hundreds of genes, some of which are linked to the control of brain development in embryos and of certain functions in adulthood (Wohlgemuth et al. 2014). In birds the gene is expressed in the same basic areas of the brain as in humans: basal nuclei, cerebellum, thalamus and cortex or equivalent regions (pallium) in nonmammalian species. All species of birds have a similar version of Foxp2. The protein of some species of bird is $98 \%$ similar to ours, differing only in 8 amino acids. It has been proposed that the capacity for communication using song arose independently on three occasions in three groups of distant relatives of birds (humming birds, parrots, songbirds) during their evolution (Sibley y Ahlquist 1990). If this supposition is correct, then evolution may also have left its mark on the sequences of Foxp2 in songbirds, humming birds and parrots, as was the case with the human Foxp2 gene.

The introduction of the non-functional Foxp2 gene from the KE family into mice stops the protein from functioning. The mice with two copies of the gene have a lower longevity, characteristic motor disorders, problems of growth and small cerebellums. Mice with just one normal copy of the gene (as is the case of the KE human family) appear to be healthy and can produce sounds. But they have difficulties with acquiring new motor skills such as learning to run rapidly in the laboratory experimental wheel (Fisher \& Scharff 2009). 
The link between FOXP2 and neuro-motor coordination was confirmed in bats. Considerable diversity was found when sequencing the gene in 13 species of bats, which is surprising given the scarce variation between the different species. This has been attributed to the fact that some bats have echo-location. The different types correspond to different sonar navigation systems. They also require more sensory motor neurons to coordinate while flying and need to be able to adjust the ultrasonic pulses they emit every few milliseconds so as to receive them as they fly and interpret tchem correctly. This may be the reason for the differentiation in echo-location in cetaceans, as they have very little diversity of FOXP2, and their navigation systems do not need to be as complex.

Finally, it is too simplistic (and mistaken) to believe that there is only one gene that affects language. On the contrary, it seems reasonable to think that genes act in an interrelated manner, and that the alteration of one gene responsible for more general aspects can deactivate other more specific genes. Neither would it be correct to assume that there is a totally direct correlation between genes and grammatical features.

\section{Early Technology: Stone Industry and Fire}

How is it possible that hominids could survive on the open savannah, two million years ago, with ever more dependent offspring, without the species becoming extinct? And how could they colonise other continents in just one hundred thousand years? (Figure 6). No reasonable explanation can be found other than the modification of behaviour and lengthening of our organic development. Biological traces of both processes can be found in modern humans: a genetic program that lengthens our Growth and Development more than in any other primate; and a reorganisation of the brain that permits sophisticated psychological behaviour. For P.V. Tobias (1991) with the emergence of the genus Homo and the arrival of the H. habilis, hominid evolution attained a new level of organization that was not comparable in degree or kind with the other, so-called lower levels of organization. In proposing that the new level of organisation which man has attained had its 
onset with the habiline tool-makers who lived in Africa just over two million years ago. Speech and language gave humankind the powers to conquer the environment and to change the environment to suit their needs. They not only made stone tools and walls, but they spoke about what they were doing. I have in mind the ineluctable inference that they taught their children how to do these things, by word of mouth. That same propensity for language, in the fullness of time, was to provide a mechanism for transmitting not only information about material things, how to make tools, how to find and track animals, how to identify plants which could be eaten and which could not, which were good for ailments, where to live most comfortably and safely, but also ideas, beliefs, codes of conduct, rituals, customs and ideals (Tobias 1991, 844).

\subsection{Current interpretation of the stone-industries}

Stone industries have been found along of the African Great Rift Valley, dated at more than 2.5 million years B.P. The primitive handaxes and choppers from Sterfontein site were the first to be discovered in association with hominid remains in South Africa. Later the presence of stone tools was discovered in Olduvai Gorge (Tanzania), Koobi Fora (Kenya) and beside the Omo river. As well as at Hadar (Etiopia). Human use of Oldowan stone tools-which are believed to have been socially transmitted-has been hypothesized to have led to the evolution of teaching and language. The results of an experiment (Morgan et. al. 2014) supports the hypothesis that human reliance on stone tool-making generated selection for teaching and language, and imply that low-fidelity social transmission, such as imitation/emulation, may have contributed to the B700,000 year stasis of the Oldowan technocomplex; also, teaching or proto-language may have been pre-requisites for the appearance of Acheulean technology. This work supports a gradual evolution of language, with simple symbolic communication preceding behavioral modernity by hundreds of thousands of years (Ambrose 2001).

In the last million years, some behavioral tendencies that already existed were progressively strengthened and others appeared. The Late Middle 
Pleistocene stone tools ( 0.3 crones) indicate great behavioral progress. The 'Levallois' technique appeared in Africa 0.3 million years ago B.P. It is based on an ingenious prior elaboration of the stone working process. It is perfected later and disappears with the Neanderthals who came to identify almost with her in their Mousterian culture. In short, the first sapiens and the last erectus would have led a life similar to that of the current hunter-gatherer peoples, with only two basic differences: current humans we have accumulated more knowledge by generational transmission, and technologically solve what they would solve with physical strength judging by formidable bone strength. In those human societies there were already comparable levels of cognitive capacity and behaviors.

The conclusion is now that the genetic and cognitive basis had to be present in the genus Homo before the evolutionary split between the Neanderthal and modern human lineages (Zilhao 2007). That being so, why then the current debate about whether Neanderthals were incapable of possessing a level of symbolism comparable to that of modern humans? This can be partly explained by the inappropriate use of certain concepts such as the ones below (Zilhao 2006): 1) understanding the term "modern behaviour", with its underlying biological implications, which corresponds to "modern culture”. Modern hunter-gatherer societies could be regarded as different species because they do not have "industrial behaviour". The difference can be explained in terms of different trajectories in time, from isolation, not as the emergence of biological capacities for industrial behaviour. 2) The use of the term "modern" with the meaning of "different" in a biological sense; 3 ) using the term "biological dispersions" for what actually were or may have been "cultural dispersions"; 4) using the term "prehistoric cultures" for what were maybe just "techno-complex".

\subsection{Use of fire}

The ability to control fire was a crucial turning point in human evolution. No other animal can do it. But the question as to when hominins first developed this ability still remains. A tropical primate like the human being, with 
dependent offspring, cannot survive without fire in the African savannah, and even less so in a cold environment.

The site of Wonderwerk Cave, Northern Cape province, South Africa, provides unambiguous evidence that burning took place in the cave during the early Acheulean occupation, approximately 1.0 Ma. This is the earliest secure evidence for burning in an archaeological context. However, there is other convincing evidence of even earlier burning in a number of sites in Koobi Fora, at Lake Turkana (Kenya), dated to 1.6 crones (Wolpoff 1999, 507-508). By that time, fire may have been used intermittently in Africa as long ago as 1.5 million years B. P., but its regular use at this time seems very improbable.

Early humans must have observed such wildfire. Fire has a fascination even to sophisticated modern people, and perhaps this fascination has always existed in the current humans. Perhaps an early human approached a natural fire fearfully but curiously. He or she could easily have managed to hold a burning twig and, touching that twig to a shrub or tuft of grass, could have multiplied the flame. One can imagine the sense of power and wonder that this person would have experienced. The warmth of fire is felt quickly, and even the inexperienced Homo erectus must have imagined its usefulness in a cold cave and attempted to bring the fire indoors.

If the use of fire has its origins in Africa, then there is little doubt that it was promoted outside the continent as an adaptation to colder latitudes such as Europe and China (Figure 6). The basic utility of fire is as a defence against cold and predators at night (all animals, without exception, flee from fire). Its use also had three other repercussions: 1) on modification of the environment. It most likely preceded clothing as a defence against the cold and to maintain body temperature. The Australian aborigines, who sleep in deserts at low temperatures, do not cover themselves, they move closer to the fire, which they transport constantly. It is essential to keep a fire burning as a defence against predators. It means independence from sunlight for illumination. It can be used as a way to prepare wood instruments (hardening points). 2) In human morphology. Softened foodstuffs allowed for increased variety and ways of consuming them. Mastication times were reduced, 
and other changes were made possible such as gracilization of the jaws, reduction of the size of molars and robustness of the masticating muscles. The ends of the brow ridge were also reduced, the forehead expanded, and the thickness of the parietal lobe was reduced. 3) In behaviour. It is highly likely, although speculative, that reflex inhibition was boosted, or rather, the control of the tendency to immediately respond to stimuli, as animals do. Handling fire requires attentions and had an influence on forced changes that took place in the brain. Handling fire would also have had an effect on greater communicative efforts and therefore on language. Keeping a fire leads makes for greater social complexity, as it requires counter-cyclical measures to watch over it at night in case of predators, on which group survival depends. With Homo sapiens, its influence would later extend by increasing free time for productive activities, and the relations between fire, religion and primitive art, which always appears at the back of caves where access is impossible without a light source.

The capacity of symbolic thought that is deduced from the use of stone tools and the use of fire indicates how far prehistoric man had entered Culture. Hallowell (1961) wrote_

The psychological basis of culture lies not only in a capacity for highly complex forms of learning but in a capacity for transcending what is learned, a potentiality for innovation, creativity, reorganization and change.

Erich Fromm (1959) wrote that man:

is driven by the urge to transcend the role of the creature," and that "he transcends the separateness of his individual existence by becoming part of somebody or something bigger than himself" (quoted by Dobzhansky 1971, 45).

\section{Evolution of the Family}

Human reproductive strategy was crucial to the survival of increasingly dependent offspring. An enormous investment in care is a distinctive feature of the human lineage, which we shall analyse below. 
Table 1. Registered longevity (average of males and females), body mass, and encephalization ratio in the first hominids and in hominoids (From Tobias 1997)

\begin{tabular}{lccc}
\multicolumn{1}{c}{ Extinct species } & $\begin{array}{c}\text { Registered longevity } \\
\text { (age of death in years) }\end{array}$ & $\begin{array}{c}\text { Body mass } \\
\text { in kgs. }\end{array}$ & $\begin{array}{c}\text { Jerison } \\
\text { EQ 67 }\end{array}$ \\
\hline A. afarensis & - & 37 & 3.1 \\
A. africanus & $-20(35 \%$ o.f.) & 35 & 3.4 \\
A. boisei & - & 48 & 3.0 \\
A. robustus & $<20$ (60\% o.f.) & 44 & 3.5 \\
H. habilis & $<20$ (73\% o.f.) & 48 & 4.0 \\
H. erectus & & 53 & 5.5 \\
(Asia and Africa) & & & \\
H. erectus pekinensis & & 53 & 6.1 \\
& & & \\
Current species & 40 & 140 & 1.7 \\
\hline Gorilla Gorilla & 31 & 19 & 2.3 \\
Papio cynochephalus & 32 & 6 & 2.4 \\
Hylobathes lar & 46 & 53 & 2.5 \\
Pongo pygmeus & 46 & 45 & 2.6 \\
Pan troglodytes & 100 & 57 & 7.6 \\
Homo sapiens & & & \\
\hline
\end{tabular}

Available demographic has revealed that the percentage of $A$. africanus who died before they were anatomically mature (35\%) is well below the percentage values for A. robustus (60.5\%), H. habilis (73\%) and H. erectus (73\%) (Tobias 1999). This leads us to the conclusion that the large-brained $H$. habilis and $H$. erectus had shorter average life spans that the small-brained $A$. africanus, which is linked to the climate change that took place 2.5 mya. On the other hand, the 'high risk' H. habilis demographic mode compared to the 'low risk' A. africanus pattern can also be seen as encephalization in hominins (although not necessarily in monkeys and baboons or in cetaceans) and was a highly vulnerable process. Tobias has tested this hypothesis, comparing data from both the demographic pattern and encephalization (Table 1). The conclusions are that 1) Chimpanzees, with only one third of the brain 
size of modern humans, are less encephalized than Australopithecines 2) $H$. habilis was appreciably more encephalized than A. africanus, and had reached or surpassed the $50 \%$ mark in relation to mean encephalization values of modern humans; 3) the differential demographic patterns are linked to the climate change of 2.5 mya; 4 ) the different demographic patterns would appear to be due to the intensity of encephalizing pressures, and the increased metabolic burden of larger brains was a restrictive handicap.

H. habilis survived in the open African savannah, a very dangerous place for a biped. It may seem paradoxical that $73 \%$ of $H$. habilis died at a subadult age, a percentage that leads to extinction. The explanation would be on the one hand that a demographic strategy had commenced: reduced birth spacing. There were more babies: some would die prematurely and others would survive. On the other hand, longer infancies would start to be the norm, which would have reduced reproductive tension.

In conclusion, the new data indicate that in the increasingly encephalized 'early Homo' there was an increase in mortality predators mean danger for land-based life, particularly for the young. Moreover, many serious tropical diseases are endemic in the areas of hominid evolution. Given that such high mortality can only lead to extinction and the surprising fact that this did not occur, the conclusion is that birth spacing had already shortened in early Homo. That is, mortality increased but there was also survival in a high-risk environment.

The brain organisation of $H$. habilis, along with the demographic success described above, enables the geographical expansion documented in the archaeological record 1.9 million years ago to be understood. Finds of so-called 'early Homo' have been discovered from Africa to the Caucasus (Dmanisi), and from India to Insulindia (Figure 6).

\subsection{Sex and estrous}

The chimpanzee is probably our best model for the demographic strategy of hominoids before the origin of hominids. The sexual maturity of a chimpanzee occurs at 10 years and average birth spacing is five and a half years, apparently because of the long period of female frigidity. The gestation 
period is thirty-four weeks and infant dependency is about six years. The young are suckled for four and a half to six years, and are carried by the mother for four years, hanging on tenaciously. Falls are not infrequent as the mother moves among the trees and the young are often injured or killed. If the mother dies, the young die when under 5. Adoption does not usually take place, as other females are occupied. It should be pointed out that males play no part in parental care, not even in providing food; this is a purely matrifocal society.

In hominids, however, an important result of being land based was that parenting was much less stressful for females, with the young living in 'nests' at the living site. Parenting could be shared among mothers, which was very important in such hazardous times, for it often led to orphans being cared for after the mother's death. However, as Lovejoy stresses, the most significant demographic advantage was to become patent when the males began to share in parental care. Lovejoy (1981) pointed out the following supporting factors:

Firstly, with diminished maternal stress and with the improved nourishment provided by food-sharing, the females gradually became continuously sexually receptive, as occurs for Homo today, even through the menstrual cycle. As a result, birth spacing was reduced. This receptivity results from the oestrogen level, which continues through the menstrual cycle with highest values before menstruation and dropping to about onethird of this level afterwards.

Secondly, with the erect posture there was exposure of the epigamic features of both sexes - penis, mammae, pubic and axillary hair - together with the secretions by the ecocrine and apocrine glands of sexually attractive odors. Moreover, the hair covering of the body regressed, lending the skin a more attractive appearance.

Thirdly, association in food-sharing and child care was to lead to still closer association in pair-bonding, with both partners sharing in the parenting of the young, the result of which was to be improved survivorship. However, it is probably a mistake to assume that the males realized their biological paternity of the offspring through pair-bonding; sexual intercourse 
was a reward in itself. With the reduction of birth spacing to an average of two and a half years, a natural average for Homo today, there would be several siblings in the human family at any one time. With Homo the dependence of the child was to be extended to up to fifteen years or more.

These changes in reproductive performance were probably dependent on the limbic system. For example, with decreased maternal stress there would have been a continuous high level of blood oestrogen due to hypothalamic production of the folliclestimulating hormone $(\mathrm{FSH})$ releasing factor, which causes pituitary secretion of FSH, which activates the oestrogen production of the ovaries. On the other hand, the size index for the septum increased from 2.09 for simians to 5.45 for Homo, and for the lateral amygdala there was an increase from 3.05 for simians to 6.02 for Homo. In contrast, the size index of the nucleus involved in rage and aggression, the medial amygdala, underwent a smaller increase, from 1.30 for simians to 2.52 for Homo. It would appear that during evolution natural selection had given rise to a genetic code that led ontogenetically to an increase in the nuclei involved in pleasure and friendliness compared to the nucleus involved in anger and aggression. Furthermore, in hominid evolution there was a considerable increase in the limbic system nuclei that are involved in pleasurable feelings and friendly behavior Epigamic displays also excite the libido, especially by visual, tactile, and olfactory sensing. Hence there is reinforcement of the male-female bonding that will eventually be refined into the sentiment of love (Eccles 1989).

\subsection{The Hip and Birth}

The increase in selective pressure on an increasingly encephalized foetus came into conflict with the platypelloid pelvis of earlier hominids. Platypelloid or flat pelvis, in obstetric terms, means that the ischio-pubic index is large: 140 in Australopithecines versus a maximum of 120 in modern females. When the innominate is viewed laterally, the pubis is seen, in upper view, as a continuous straight extension of the ilium, while in modern humans the pubis is more perpendicular to the ilium. According to Tague and Lovejoy (1985), with the hyperplatypelloid pelvis of A.L. 288(1) 'Lucy', the 
australopithecine foetus may have entered the pelvis in transverse diameter, a unique trait among primates, as a result of selection for improved visceral support in a bipedal animal.

Modern human young are extremely undeveloped at birth compared with infants of other primates' species (Figure 7). It has been proposed that by 1.5 mya, pelvic size limited prenatal brain growth (Martin 1983). Two changes may have allowed encephalization to continue: the fact that human infants had a smaller percentage of adult brain size at birth (secondary altriciality); or the fact that birth occurred at a relatively earlier time in foetal development. The only other possibility would be pelvis shape alteration and the change in the mechanism of birth (Rosenberg \& Trevathan 1995/96).

Table 2. Brain size at birth and in adults, in apes, Homo (current) and fossil hominids (deduced) (From Tobias 1981)

\begin{tabular}{lcccc} 
& $\begin{array}{c}\text { Average } \\
\text { brain size } \\
\text { in the adult }\end{array}$ & $\begin{array}{c}\text { Brain size } \\
\text { at birth }\end{array}$ & $\begin{array}{c}\text { Percentage } \\
\text { of the size } \\
\text { with respect } \\
\text { to the adult } \\
\text { cc }\end{array}$ & $\begin{array}{c}\text { Estimated brain } \\
\text { size if there if } \\
\text { birth is not } \\
\text { anticipated } \\
\text { cc }\end{array}$ \\
\hline Ape & 480 & 300 & 60 & \\
Australopithecus & 480 & 300 & 60 & 300 \\
H. habilis & 646 & 300 & 46 & 390 \\
$\begin{array}{l}\text { H. erectus } \\
\text { (Asia and Africa) }\end{array}$ & 890 & 300 & 35 & 530 \\
H. erectus pekinensis & 1043 & 300 & 29 & 625 \\
H. sapiens & 1344 & 350 & 26 & 810 \\
\hline
\end{tabular}

Tobias (1981) has demonstrated on obstetrical grounds the necessity for this progressive predating of parturition. He proposes that, in accordance with the evolutionary increase in brain size, there must have been earlier births to ensure that the foetal skull was not out of proportion to the size of the birth canal through the female pelvis. Table 2 shows average mea- 
surements for brains at birth and adult brains for ape and Homo. Assuming that the modern ape is an approximate model for our hominoid ancestors, it is surprising that brain size at birth changed so little in hominid evolution (300-350 cc.) despite the three-fold increase in the respective adult brains.

If the brain at birth had remained at 60 per cent of the adult size (the ape proportion) in hominid evolution, it would have been impossibly large, as shown in the fourth column of Table 2. It was an evolutionary need for parturition to take place at progressively earlier stages of foetal brain development.

\section{Human Ontogeny}

The vast majority of mammals pass from infancy to adulthood without going through any intermediate stages, and in this stage the pace of growth decreases gradually. However, in the case of social mammals, such as lions, dogs, wolves, elephants and primates, puberty is postponed and a new, juvenile, state is introduced, with features that differentiate it in terms of growth and of behaviour from infant and adults stages. The juvenile stage consists of those who are no longer dependent on their mothers for survival. They are no longer defenceless but they still require attention from adults. The appearance of such a stage and its duration in the life cycle is a response to basic adaptation related to locomotion, food acquisition and reproduction.

By contrast, we humans have two more states than other primates and mammals: childhood and adolescence. Human Growth and Development consists of five stages: infancy, childhood, youth, adolescence and adulthood, each one of which can be identified from a biological and behavioural perspective, and which are related to the growth rate, nutrition and sexual conduct.

B. Bogin (2003) proposed a framework (Figure 8) that effectively summarises the evolution of different stages in hominids, although it is an approximation based on fossil evidence, with the exception of modern humans.

A. afarensis is a hominid that still shares many characteristics with the chimpanzee, such as a similar sized brain in adulthood, of about $400 \mathrm{cc}$. and 
a dental development model that is indistinguishable from apes. For this reason it is represented in Figure 8 with the empirical data of the chimpanzee, infancy, youth and adulthood, in which infancy is the breastfeeding period, the juvenile stage represents a period of nutritional self-reliance before the onset of sexual maturity, and adulthood commences with puberty and sexual maturity. In the case of $A$. africanus (Table 2), who has a greater brain capacity but similar encephalization coefficients, it is not incorrect to consider that the foetal stage and infancy may have been prolonged by one year, as observed in other mammals.

The same situation can be applied to $H$. habilis. Its increased brain size (610-800cc.) may have been brought about by longer foetal and infant periods, and possibly even extended periods as juveniles. However, the growth of the femur is different from the australopithecine model and looks more similar to that of $H$. erectus, thus making it likely that it appeared after a brief period of infancy. The appearance of infancy with $H$. habilis also matches the demographic data available today (Tobias 1977 y 1999), which would have reduced reproductive tension. A longer infancy favours the chances of survival of offspring young since female primates, including humans, cannot procreate a second child if they are still breastfeeding the first one.

The increase in brain size to $850-900 \mathrm{cc}$. in the first $H$. erectus is probably a reflection of a longer juvenile stage. On the other hand, the Figure 8 shows that infancy progressively decreased as the juvenile period grew longer. This should have given $H$. erectus a reproductive advantage over other hominids, and was very probably associated with their geographical expansion 1.8 mya ago.

There is evidence that the first $H$. erectus did not experience the 'growth spurt'. Analysis of the teeth and skeleton, of 1.8 crones, KNM-WT 15000, the youngster of Lake Turkana, who would have had a dental age of 14.05 when he died, indicates that he was a young adult given that he had reached most of his bodily development at that age. He would be 11.4 years old when compared to a chimpanzee, and between 18 and 21 (Smith 1993) when compared to a human. It can therefore be deduced that adolescence would not yet have appeared amongst $H$. erectus. 
There was an even greater growth in the brain size of the final H. erectus, up to 1100 cc., which implies a longer period of infancy and the appearance of adolescence. The increase in technological industry (more complex tools, use of fire) and a more complex social organisation made the adolescent stage necessary.

With the arrival of Homo sapiens the current expansions of the stages of childhood and adolescence appeared.

\section{On Human Altruism}

The term altruism was coined by the positivist philosopher, Auguste Comte, in the mid-19th century, to refer to a particular type of moral conduct in which a person tries to do good to others without thinking of any benefit that they might obtain.

Biological determinists (socio-biology) see in this conduct an effect of "group selection", in other words, some individuals receive genes to sacrifice themselves for others. E. O. Wilson distinguishes between extreme altruism, the sacrifice made by a mother for her offspring, with a powerful emotional component, y ordinary altruism, which would be selfish, or rather, that behind the act there is the expectation of a reward.

Eccles (1989) distinguishes between pseudo-altruism, that of animals, from real altruism, which is characterised by its intentionality, although it may be automatic when it is carried out, and by planning with regard to the interests of the other person or persons. In fact, there is no altruism of this nature in any animal, although there was in the human past, as with the case of the "toothless" Homo erectus found in the site of Dmanisi, of 1.77 crones, who needed to be fed and cared for. Behaviour of this nature exceeds that practiced by non-human primates. Other examples can be seen in Neanderthals, where bone scars indicate wounds from severe injuries in individuals who then survived, which shows that they were cared for.

Campbell (1998) distinguishes between bio-altruism, of animals, and reciprocal altruism, taken from Hamilton and Trivers. This type of altruism refers to the generalised feeling that any good action (e.g., saving someone else's life) generates a tacit debt in the beneficiary, who is then indebted 
to the saviour, in the future, before third parties. There would be a relation between this and strong social rejection caused by fraud and betrayal, in other words, by breaching agreements. The degree of genetic imprinting that reciprocal altruism might possess is unknown, but frustrating daily experience shows us that there is no evident biological determinism. At present, as we mentioned above, the interaction of the environment with the epigenome substantially modifies a biological determinism of altruism.

What Dobzhansky (1956) called The biological basis of human freedom' is now of greater relevance. Human beings show behaviours that are not adaptive, and are even suicidal, which is a rational act made possible by brain evolution, which enable conducts not associated with emotions to be planned and put into practice. This is the basis of human freedom.

It is useful to observe altruistic behaviour in children. Although learning by imitation is fundamental to accumulating culture, chimpanzees also learn by observing a model and, however, they have not accumulated culture. Experiments suggest that chimpanzees are more inclined to emulate, or to learn to obtain the desired outcome, than to imitate, where the precise means are copied. For example, children imitate unrewarded actions presented by an adult model, while chimpanzees rapidly eliminate actions that do not lead to a reward being obtained. Other studies have shown that this is a constant difference in humans when compared to chimpanzees in social learning. The human tendency towards altruistic behaviour, the propensity for imitation without reward is very unusual in nature. On the other hand, experiments show that children easily imitate the altruistic behaviour of others, which has not been observed in the other primates. Children not only imitate models of behaviour but also incorporate the emotional reaction that doing things any other way is bad.

Human social learning includes an exceptional capacity to focus on actions instead of objectives. On the other hand, chimpanzees imitate behaviours that give immediate rewards, but are slow to foresee new behaviours that can provide even greater benefits.

It has been proposed that human culture is conceptually different from the traditions of animals in that there are three components that are 
universally present in all human societies: 1) socially learnt techniques 2) socially learnt rules of conduct (agreements, norms, laws) that operate via rewards and punishments; 3) symbolic and emotional reinforcement of a system of specific rules (ritual, moral, religion, race). The second and third components have not been described in non-human animals. Such cognitive capacities are decisive in understanding the formidable and distinctive human capacity to transmit culture via teaching.

\section{Conclusions}

Humans are very a peculiar species, unique, provided with a highly developed capacity for symbolic thinking, a semantic-phonetic language, and a complex corpus of traditions known as Culture, which is the exclusive property of humankind (Holloway 1969).

From a biological perspective, human culture is distinguished from other animal species by an enormous investment in social learning, the result of which is an enormous accumulation of extra-somatically stored information. This information system is the prevailing force of adaptation in human beings, but not in other living beings. Humans also possess a complex system of symbolic communication that enables information about events to be transferred using articulated language.

There is a Great Breach that separates humanity in the place it occupies in Nature, sometimes it seems to become smaller with the impressive advances made in science. But no other animal has attained the exclusive properties of humanity in terms of Rationality and Ethics, which can only come from Freedom, and which are unthinkable without it. The intentional use of fire, which took place at least a million years ago, provides the unshakeable guarantee that these three characteristics already existed back then, and probably before when they excavated inside caverns, since environmental erosion has wiped away most of the traces of fire from the habitats of ancient humanity.

A human being that more than a million years ago transformed all kinds of materials (volcanic stone, bamboo) into objects or utensils; who made 
boats, huts, stockades, lake dwellings; the very same being already showed a capacity for abstract concepts (in a philosophical sense). Other features should not be forgotten, such as the viability of offspring, of maternal love, of group cooperation and therefore of the inevitable appearance of Ethics as social beings that deeply depend on each other for their survival.

This Great Breach is the vacuum between the power of human thought and other primates, including chimpanzees. What separates us from other animals are:

1) Independence of feelings (remodelling of the limbic system).

2) Self-consciousness: sense of I and the others.

3) Abstraction and Ethics (moral values). Freedom.

4) Symbolism: conceptual (mathematic) and of the senses (art).

5) Futurity: planning for the future, feeling of death.

6) Combinations and permutations: mathematics, grammar (syntax), articulated language.

7) Artistic feelings and religiosity.

8) Sense of humour and irony.

Dobzhansky $(1967,58)$ comments:

The biological revolution has transcended itself in the human "revolution". A new level or dimension has been reached. The light of the human spirit has begun to shine. The humanum is born.

Since these words were written, it has become clear that things did not happen all at once, as in a sort of revolution. More than a million years ago (and perhaps several millions) elapsed between the attainment of uprightness and the making of the first stone tools. Hundreds of thousands of years might have separated the earliest stone tools and the birth of language.

$H$. habilis would be human in all the potentialities of the human spirit although not yet fully manifested. Rather, Tobias suggested (1991, 845):

[...] the concept should be applied to the birth of the genus Homo, the rise of H. habilis, in the closing phase of the Pliocene Epoch. This was the first hominid who substantially distanced himself from his animality; who developed a whole new 
tool-kit for survival; who at last employed those emancipated hands to wrest a variety of implements from tough and intractable materials; who built a shelter behind rock walling; and who spoke about what he was doing. That moment of transmutation deserves, perhaps demands, to be rated as a transcendence. A new integrative plane, a new level of organization, was attained. By taking this great step forward, Homo habilis was enabled by its cerebral revolution to attain a new mode of evolution, as an articulate, languagebound, culture-dependent hominid.

\section{References}

Ambrose, Stanley H. 2001. "Paleolithic Technology and Human Evolution.” Science 291: 1748-1753.

Berna, Francesko, Paul Goldberg, Liora K. Horwitz, James Brink, Sharon Holt, Marion Bamford, Michael Chazan. 2012. "Microstratigraphic evidence of in situ fire in the Acheulian strata of Wonderwek Cave, Northern Cape province, South Africa”. PNAS 109 (20), E1215-E1220; https://doi.org/10.1073/pnas.1117620109

Bolhuisn, Johan J, Kazuo Okanoya, Constance Scharff. 2010. “Twitter evolution: converging mechanisms in birdsong and human speech”. Nature Reviews Neuroscience 11: 747-759.

Bogin, Barry. 1997. "Evolutionary Hypotheses for Human Childhood”. Yearbook of Physical Anthropology vol. 40: 63-89.

Bogin, Barry. 2003. "The human patterns of groth and development in paleontological perspective". In Patterns of Growth and Development in the Genus Homo, eds. Jennifer L. Thompson et al., 15-44. Cambridge: Cambridge University Press.

Campbell, Bernard G. 1998. Human Evolution, Fourth Edition. Chicago: Aldine Publishing Comp.

Dobzhansky, Theodosius. 1956. The biological basis of human freedom. New York: Columbia University Press.

Dobzhansky, Theodosius. 1962. Mankind evolving. The evolution of Human Species. New Haven and London: Yale University Press.

Dobzhansky, Theodosius. 1967. The biology of the ultimate concern. Collins, London: The Fontana Library.

Eccles, John C. 1989. Evolution of the Brain: Creation of the Self. New York: Routledge.

Fisher, Simon \& Constance Scharff. 2009. “FOPP2 as a molecular window into speech and language". Trends in Genetics 25(4): 166-177.

Fromm, Erich. 1959. "Value, Psychology, and human existence”. In New Knowledge of human values, ed. Abraham Harold Maslow, 151-164. New York: Harper \& Row. 
Green, Richard E, Johannes Krause, Adrian W. Briggs, Tomislav Maricic, Stenzel Udo, Martin Kircher, Nick Patterson et al. (2010) "A draft sequence of the Neanderthal genome”. Science 328: 710-722.

Hallowell, Irving A. 1961. “The protocultural foundations of human adaptation”. In Social Life of early Man, ed. Sherwood Larned Washburn, 236-255. New York: Werner Green Foundation.

Holloway, Ralph L. 1969. “Culture: a human domain”. Current Anthropology 10(4):395-412.

Holloway, Ralph L. 1995. "Toward a synthetic theory of human brain evolution". In Origins of the Human Brain, eds. Jean-Pierre Changeux and Jean Chavaillon, 42-54. Oxford: Oxford Clarendon Press.

Holloway, Ralph L. 2009. “Brain Fossils: Endocasts”. In Encyclopedia of Neuroscience, ed. Larry R. Squire, volume 2, 353-361. Oxford: Oxford Academic Press.

Lévi-Strauss, Claude. 1949. The Elementary Structures of Kinship. London: Eyre \& Spottiswoode (1969 ed.).

Malinowsky, Bronislaw. 1926. Crime and costum in savage society. London: Routledge \& Kegan Paul (1966).

Marino, Lori. 1996. “What Can Dophins Tell Us About Primate Evolution?” Evolutionary Anthropology 5:81-85.

Martin, Robert D. 1983. Human brain evolution in an ecological context. Fiftysecond James Arthur lecture on the evolution of the human brain. New York: American Museum of Natural History.

Mendez, Fernando L., Thomas Krahn, Bonnie Schrack et al. (2013) “An African American paternal lineage adds an extremely ancient root to the Y Chromosome phylogenetic tree”. American Journal of Human Genetics 92:454-459.

Morgan, Lewis Henry. 1877. Ancient Society. London: MacMillan \& Company

Morgan, Thomas J.H., Natalie Uomini, Luke Rendell et al. 2015. “Experimental evidence for the co-evolution of hominin tool-making teaching and language”. Nature Communications 6:6029 doi: 10.1038/ncomms7029.

Reich, David, Nick Patterson, Svante Pääbo, Mark Stoneking et al. 2011. "Denisova admixture and the First Modern Human dispersals into Southeast Asia and Oceania”. American Journal of Human Genetics 89: 516-528.

Roebroeks, Wil, Paola Vila. 2011. “On the earliest evidence for habitual use of fire in Europe”. PNAS 108(13):5209-5214.

Rosemberg, Karen, Wenda Trevathan. 1995. "Bipedalism and human birth: the obstetrical dilemma revisited”. Evolutionary Anthropology 4(5):161-168.

Sankararaman, Sriram, Nick Patterson, Heng LI, Svante Pääbo. 2012. “The date of interbreeding between Neandertals and Modern Humans”. Plos Genetics 8 (10): e1002947. 
Scally, Aylwyn, Richard Durbin. 2012. "Revising the human mutation rate: implications for understanding human evolution”. Nature Reviews 13:744-752.

Schlebusch, Carina M., Pontus Skoglund, Per Sjodin et al. 2012. “Genomic variation in seven Khoe-San groups reveals adaptation and complex African history”. Science 338(6105):374-379.

Sibley, Charles \& Jon Alquist. 1990. Phylogency and classification of birds: a study in molecular evolution. New Haven, Connecticut: Yale University Press.

Tague, Robert G. \& Owen C. Lovejoy 1986. “The obstetric pelvis of A.L. 288(1) Lucy”. Journal of Human Evolution 15:237-255.

Tanner, James M. 1977. “Human growth and constitution”. In Human Biology, eds. Gordon A. Harrison, Joseph Sidney Weiner, James Mourilyan Tanner and Nigel A. Barnicot (Second edition), 301-384. Oxford: Oxford University Press.

Tobias, Philip V. 1981. Evolution of the Human brain, Intellect and Spirit. Andrew Abbie Memorial Lecture. Adelaide: Adelaide University Press.

Tobias, Philip V. 1987. “The brain of Homo habilis: A new level of organization in cerebral evolution”. Journal of Human Evolution 16:741-761.

Tobias, Philip V. 1991. The Skulls, Endocasts and Teeth of Homo habilis. Olduvai Gorge, vol IV. Cambridge: Cambridge University Press.

Tobias, Philip V. 1997. "Evolution of the Brain Size, Morphological Restructuring and Longevity in Early Hominids”. In Principles of Neural Aging, eds. Sergio U. Dani, Akira Hori, and Gerhard F. Walter, 153-174. Amsterdam: Elsevier.

Tobias, Philip V. 1998. “Evidence for Early Beginnings of Spoken Language. En 'The Origins of the Speech”'. Cambridge Archaeological Journal 8(1):72-78.

Tobias, Philip V. 1999. „Dating, demography, ecology and encephalization in relation to human evolution”. South African Journal of Science 95:189-193.

Trinkaus, Erek. 2007. "Human evolution: Neandertal gene speaks out”. Current Biology 17(21):R917-R919.

Turbón, Daniel. 2006. La Evolución Humana. Barcelona: Ariel.

Wohlgemuth Sandra, Adam Iris, Constance Scharff. 2014. "FoxP2 in songbirds". Current opinion in Neurobiology 28:86-93.

Wolpoff, Milford. 1999. Paleoanthropology. Second Edition. New York: McGraw Hill. Zilhao, João. 2006. “Neandertals and Modern mixed, and it matters”. Evolutionary Anthropology 15:183-195.

Zilhao, João. 2007. “The emergence of the ornaments and art: an archaeological perspective on the origins of 'Behavioral Modernity'”. Journal of Archaeological Research 15:1-54. 


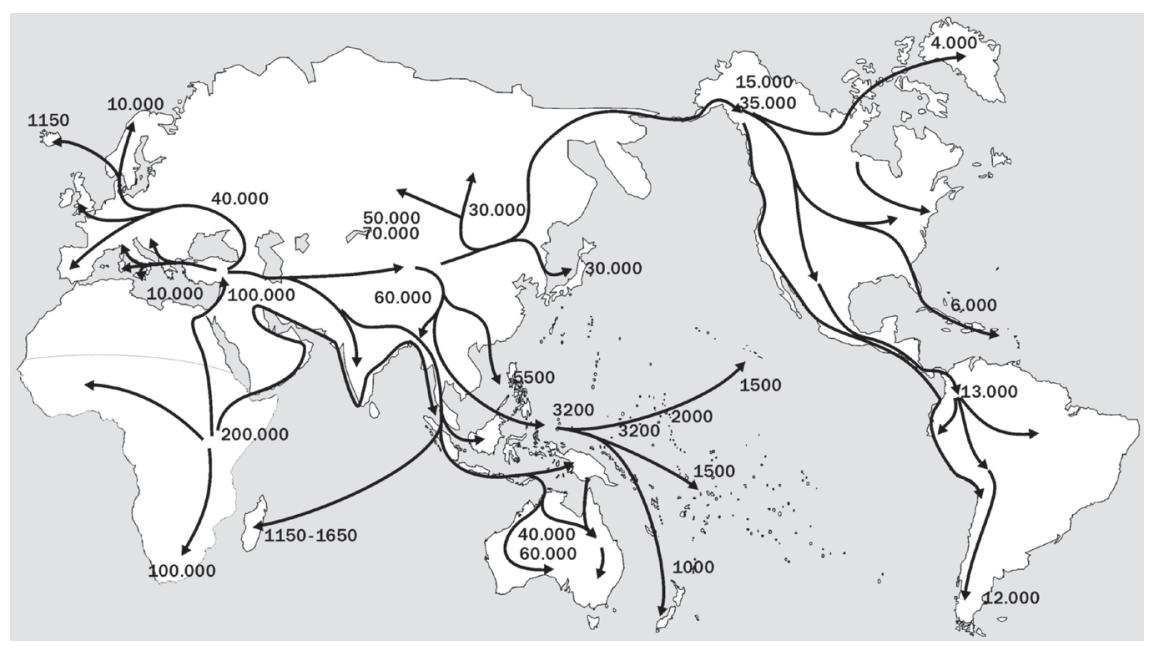

Figure 1. Routes of human migrations according to mitochondrial DNA (From Turbón 2006)

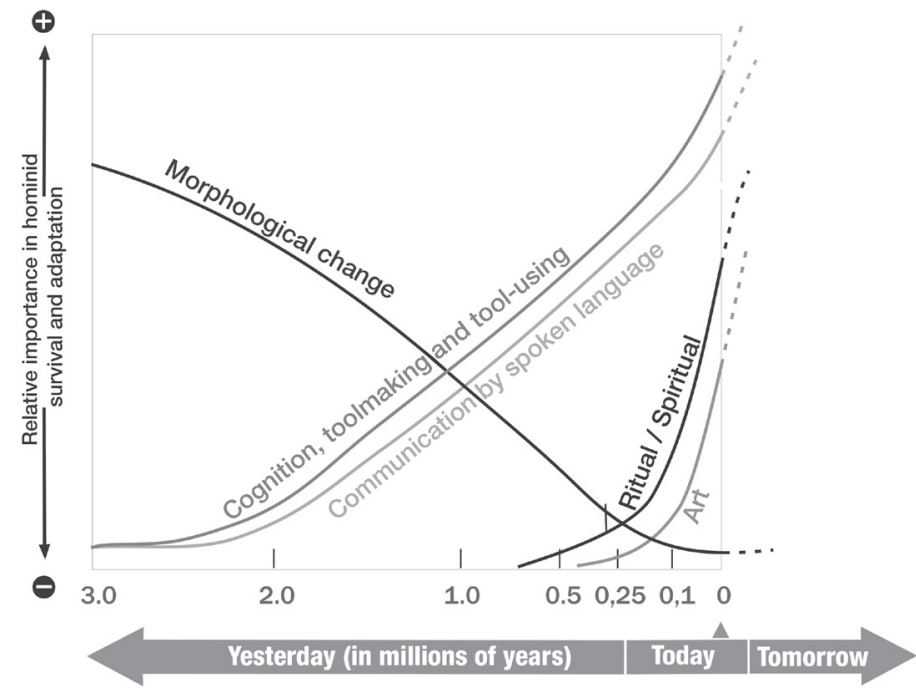

Figure 2. Time and adaptation in hominid evolution. The diagram suggests that morphological changes, which still occur, have had decreasing importance in hominid survival and adaptation, at the expense of the progressive dominance of functional, cognitive and linguistic behaviour (From Tobias 1997) 


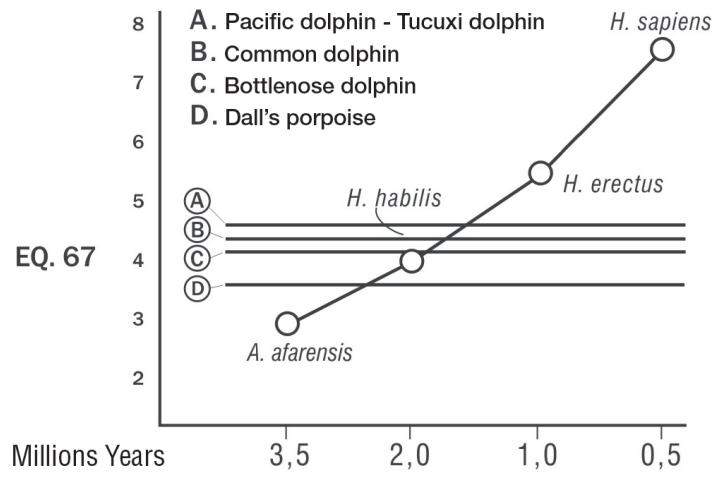

Figure 3. Approximate EQ 67 for each of the four major hominid ancestral and modern species at the time of their emergence, along with EQ67 values for four modern delphinids and one modern porpoise. With Homo habilis, man reached a level similar to that of dolphins, and, since then, he became Nature's most encephalized being (From Marino 1996) 


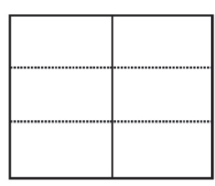

A

Size only
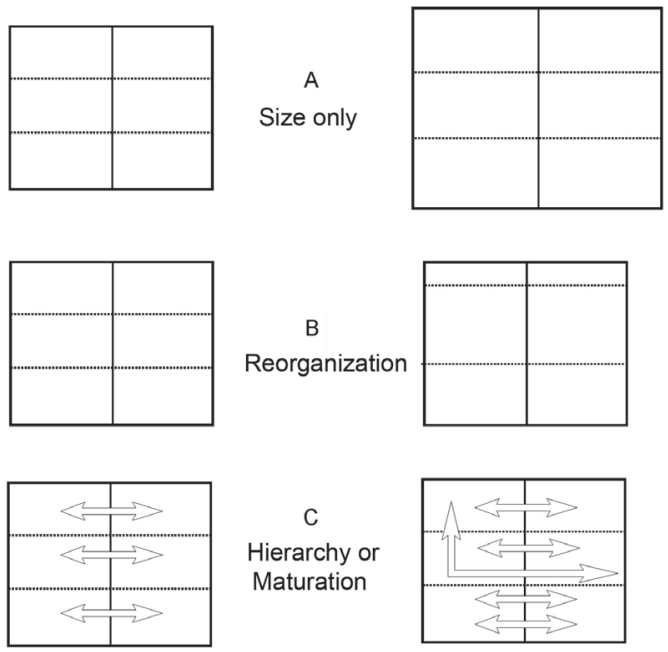

C

Hierarchy or

Maturation
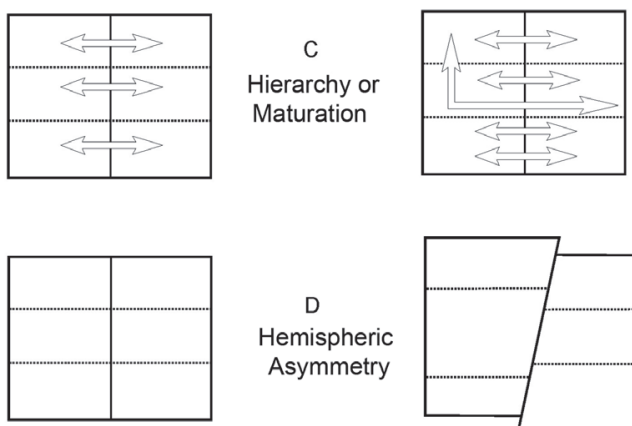

D
Hemispheric
Asymmetry
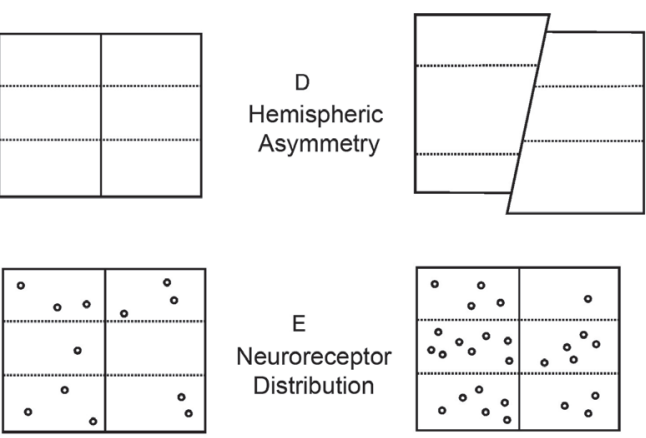

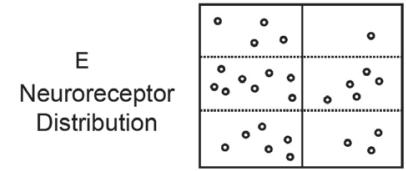

Figure 4. Some possible scenarios of brain changes in human evolution. Scenarios (b-e) suggest reorganizational changes that may not be visible on endocasts, although (b) and (d) are variably present on endocasts. (b), For example, symbolizes a reduction of primary visual striate cortex, with a posteriorly placed lunate sulcus, and (d) shows the typical torque-petalial pattern we associate with right-handedness. Neither (c) or (e) are likely to show details on the cortical surface, as the changes could be related to fiber connections, or to subcortical structures not appearing on endocasts (According to Hollloway 2009) 


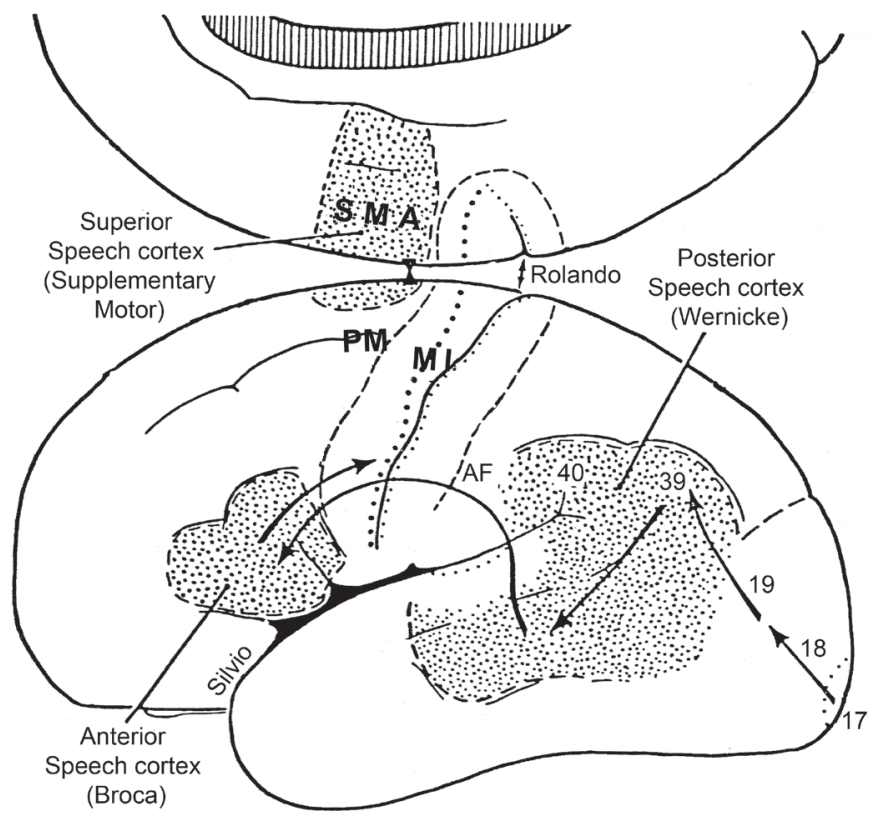

Figure 5. The left hemisphere and speech areas with frontal lobe to the left. The medial side of the hemisphere is shown as if reflected upwards. (Rolando $=$ the fissure of Rolando or the central fissure; Silvio = the fissure of Sylvius; $\mathrm{AF}=$ arcuate fasciculus.) The primary motor cortex (Ml) is shown in the precentral cortex just anterior to the central sulcus and extending deeply into it. Anterior to MI is shown the premotor cortex (PM), with the supplementary motor area (SMA), largely on the medial side of the hemisphere (From Eccles 1989; figure modified) 


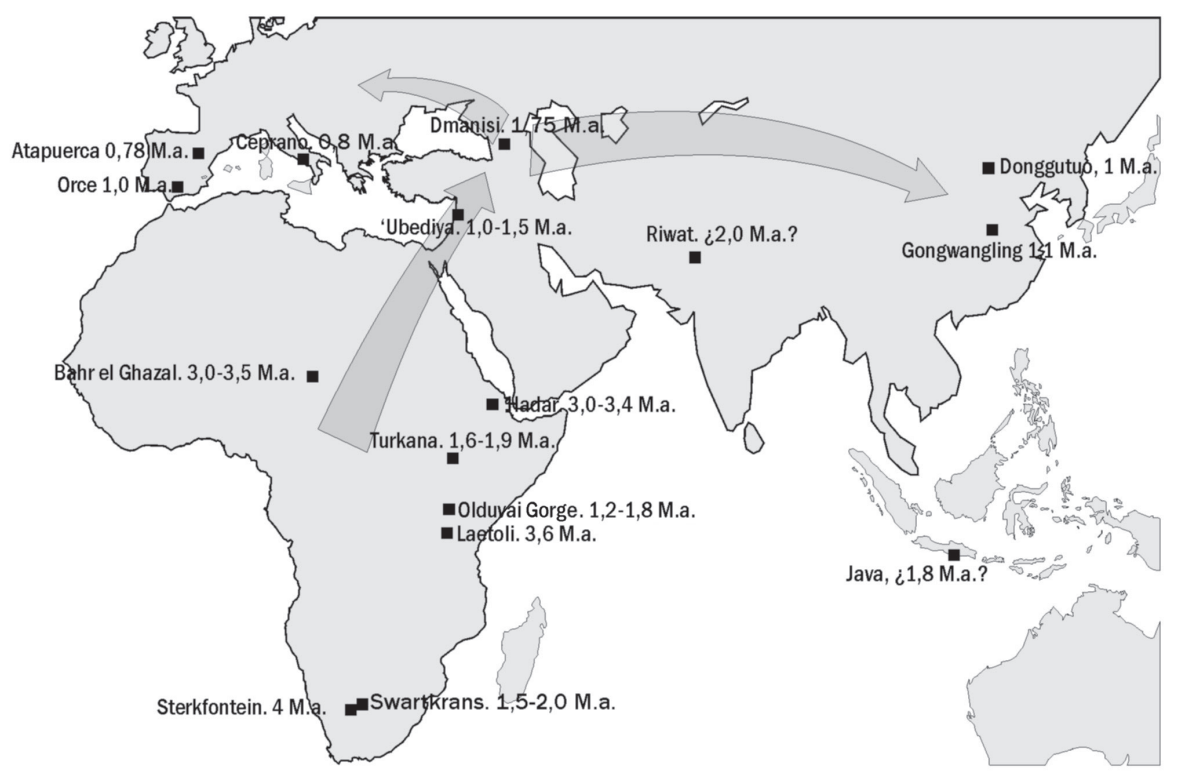

Figure 6. Migratory routes of hominids in the first expansion From Africa (1.8 million years ago) 


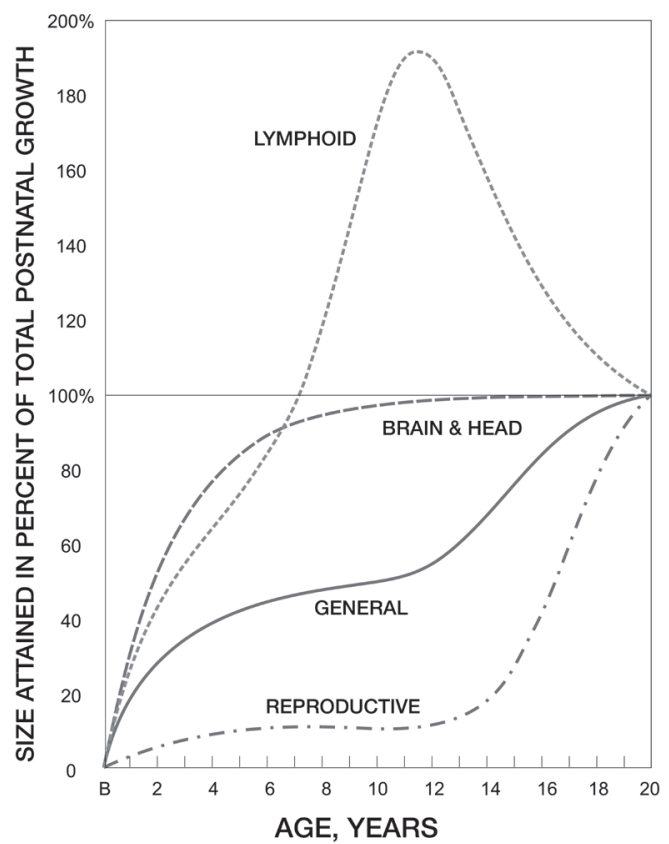

Figure 7. Growth curves of four of the main systems and parts of the human body. All curves represent the size reached during postnatal development in \%, so that the size at age 20 is 100 on the vertical scale. Note the lengthening of the growth of the body as a whole (the body except the head, the respiratory and digestive organs, the kidneys, the pulmonary and aortic trunks, the musculature and the blood volume), which barely reaches $50 \%$ before 12 year old. The reproductive system gets longer. Both elongations, with respect to other primates, imply a greater risk for the viability of the species. In the opposite direction, the human brain and head follow a completely exceptional trajectory in the animal world: they grow very rapidly after birth, reaching $80 \%$ by 4.5 years of age. Finally, the lymphatic system, which is primarily responsible for forming and activating the immune system, will not have its maximum efficacy until 6.5 years of age; it reaches its maximum in adolescence and then, probably under the influence of sexual hormones, it descends to the definitive value of the adult (From Tanner 1977) 


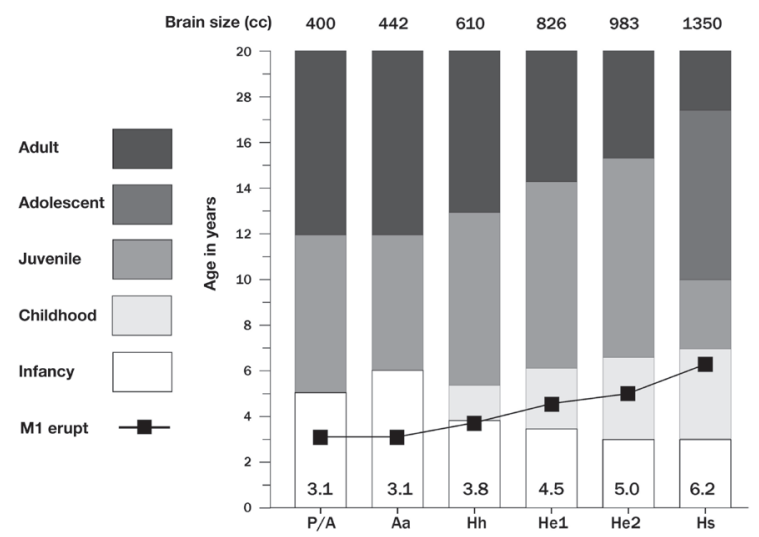

Figure 8. The evolution of the hominid life history during the first 20 years of life. Mean brain sizes are given at the top of the histogram. Mean age at eruption of the first permanent molar (M!) is given near de bottom of each histogram and is graphed across the histograms. Abbreviated nomenclature as follows: P/A Pan and Australopithecus afarensis; Aa, Australopithecus africanus; Hh, Homo habilis; He1, early Homo erectus; He2, late Homo erectus; Hs, Homo sapiens (From Bogin 1997) 
